\title{
ON THE INDEX OF A NON-FREDHOLM MODEL OPERATOR
}

\author{
Alan Carey, Fritz Gesztesy, Galina Levitina \\ AND FEDOR SUKOCHEV
}

Abstract. Let $\{A(t)\}_{t \in \mathbb{R}}$ be a path of self-adjoint Fredholm operators in a Hilbert space $\mathscr{H}$, joining endpoints $A_{ \pm}$as $t \rightarrow \pm \infty$. Computing the index of the operator $\boldsymbol{D}_{\boldsymbol{A}}=\partial / \partial t+\boldsymbol{A}$ acting on $L^{2}(\mathbb{R} ; \mathscr{H})$, where $\boldsymbol{A}$ denotes the multiplication operator $(\boldsymbol{A} f)(t)=A(t) f(t)$ for $f \in L^{2}(\mathbb{R} ; \mathscr{H})$, and its relation to spectral flow along this path, has a long history, but it is mostly focussed on the case where the operators $A(t)$ all have purely discrete spectrum.

Introducing the operators $\boldsymbol{H}_{1}=\boldsymbol{D}_{\boldsymbol{A}}^{*} \boldsymbol{D}_{\boldsymbol{A}}$ and $\boldsymbol{H}_{2}=\boldsymbol{D}_{\boldsymbol{A}} \boldsymbol{D}_{\boldsymbol{A}}^{*}$, we consider spectral shift functions, denoted by $\xi\left(\cdot ; A_{+}, A_{-}\right)$and $\xi\left(\cdot ; \boldsymbol{H}_{2}, \boldsymbol{H}_{1}\right)$ associated with the pairs $\left(A_{+}, A_{-}\right)$and $\left(\boldsymbol{H}_{2}, \boldsymbol{H}_{1}\right)$. Under the restrictive hypotheses that $A_{+}$is a relatively trace class perturbation of $A_{-}$, a relationship between these spectral shift functions was proved in [14], for certain operators $A_{ \pm}$with essential spectrum, extending a result of Pushnitski [22]. Moreover, assuming $A_{ \pm}$to be Fredholm, the value $\xi\left(0 ; A_{-}, A_{+}\right)$was shown to represent the spectral flow along the path $\{A(t)\}_{t \in \mathbb{R}}$ while that of $\xi\left(0_{+} ; \boldsymbol{H}_{1}, \boldsymbol{H}_{2}\right)$ yields the Fredholm index of $\boldsymbol{D}_{\boldsymbol{A}}$. The fact, proved in [14], that these values of the two spectral functions are equal, resolves the index $=$ spectral flow question in this case. This relationship between spectral shift functions was generalized to non-Fredholm operators in [9] again under the relatively trace class perturbation hypothesis. In this situation it asserts that the Witten index of $\boldsymbol{D}_{\boldsymbol{A}}$, denoted by $W_{r}\left(\boldsymbol{D}_{\boldsymbol{A}}\right)$, a substitute for the Fredholm index in the absence of the Fredholm property of $\boldsymbol{D}_{\boldsymbol{A}}$, is given by

$$
W_{r}\left(\boldsymbol{D}_{\boldsymbol{A}}\right)=\xi_{L}\left(0_{+} ; \boldsymbol{H}_{2}, \boldsymbol{H}_{1}\right)=\left[\xi_{L}\left(0_{+} ; A_{+}, A_{-}\right)+\xi_{L}\left(0_{-} ; A_{+}, A_{-}\right)\right] / 2 .
$$

Here one assumes that $\xi\left(\cdot ; A_{-}, A_{+}\right)$possesses a right and left Lebesgue point at 0 denoted by $\xi_{L}\left(0_{ \pm} ; A_{+}, A_{-}\right)$(and similarly for $\xi_{L}\left(0_{+} ; \boldsymbol{H}_{2}, \boldsymbol{H}_{1}\right)$ ).

When the path $\{A(t)\}_{t \in \mathbb{R}}$ consists of differential operators, the relatively trace class perturbation assumption is violated. The simplest assumption that applies (to differential operators in $1+1$ dimensions) is to admit relatively Hilbert-Schmidt perturbations. This is not just an incremental improvement. In fact, the method we employ here to make this extension is of interest in any dimension. Moreover we consider $A_{ \pm}$which are not necessarily Fredholm and we establish that the relationships between the two spectral shift functions found in all of the previous papers [9] , [14], and [22], can be proved, even in the non-Fredholm case. The significance of our new methods is that, besides being simpler, they also allow a wide class of examples such as pseudodifferential operators in higher dimensions. Most importantly, we prove the above formula for the Witten index in the most general circumstances to date.

Mathematics subject classification (2010): Primary 47A53, 58J30; Secondary 47A10, 47A40. Keywords and phrases: Fredholm and Witten index, spectral shift function.

\section{REFERENCES}

[1] N. Anghel, The two-dimensional magnetic field problem revisited, J. Math. Phys. 31, 2091-2093 (1990).

[2] N. Aronszajn And W. F. Donoghue, On exponential representations of analytic functions in the upper half-plane with positive imaginary part, J. Analyse Math. 5, 321-388 (1956-57).

[3] M. Sh. Birman And D. R. Yafaev, The spectral shift function. The work of M. G. Krein and its further development, St. Petersburg Math. J. 4, 833-870 (1993). 
[4] D. Bollé, F. Gesztesy, H. Grosse, W. Schweiger, And B. Simon, Witten index, axial anomaly, and Krein's spectral shift function in supersymmetric quantum mechanics, J. Math. Phys. 28, 1512-1525 (1987).

[5] C. Callias, Axial anomalies and index theorems on open spaces, Commun. Math. Phys. 62, 213-234 (1978).

[6] A. Carey, F. Gesztesy, G. Levitina, D. Potapov, F. Sukochev, and D. Zanin, On index theory for non-Fredholm operators: a $(1+1)$-dimensional example, preprint, 2014, Math. Nachrichten, to appear.

[7] A. Carey, F. Gesztesy, G. Levitina, D. Potapov, F. Sukochev, and D. Zanin, Trace formulas for a $(1+1)$-dimensional model operator, preprint, 2014.

[8] A. Carey, F. Gesztesy, D. Potapov, F. Sukochev, and Y. Tomilov, A Jost-Pais-type reduction of Fredholm determinants and some applications, Integral Eq. Operator Theory 79, 389-447 (2014).

[9] A. Carey, F. Gesztesy, D. Potapov, F. Sukochev, and Y. Tomilov, On the Witten index in terms of spectral shift functions, ArXiv1404.0740, J. Analyse Math., to appear.

[10] A. Carey, H. Grosse, AND J. KaAd, Anomalies of Dirac type operators on Euclidean space, Commun. Math. Phys. 335, 445-475 (2015).

[11] A. CAREY AND J. KAAD, Topological invariance of the homological index, preprint http://arxiv.org/abs/1402.0475.

[12] R. W. CAReY And J. D. Pincus, An invariant for certain operator algebras, Proc. Nat. Acad. Sci USA 71, 1952-1956 (1974).

[13] N. Dunford And J. Schwartz, Linear operators. Part II. Spectral theory. Selfadjoint operators in Hilbert space, Wiley \& Sons, New York, 1988.

[14] F. Gesztesy, Y. Latushin, K. A. Makarov, F. Sukochev, and Y. Tomilov, The index formula and the spectral shift function for relatively trace class perturbations, Adv. Math. 227, 319420 (2011).

[15] F. Gesztesy, Y. Latushrin, F. Sukochev, And Y. Tomilov, Some operator bounds employing complex interpolation revisited, Herrnhut Proceedings, W. Arendt, R. Chill, and Y. Tomilov (eds.), Operator Theory: Advances and Applications, Birkhäuser, Springer, Basel (to appear).

[16] F. GESZTESY AND R. NICHOLS, Weak convergence of spectral shift functions for one-dimensional Schrödinger operators, Math. Nachrichten 285, 1799-1838 (2012).

[17] F. GESZTESY AND R. NiCHOLS, An abstract approach to weak convergence of spectral shift functions and applications to multi-dimensional Schrödinger operators, J. Spectral Theory 2, 225-266 (2012).

[18] F. GeSZTESy AND B. Simon, Topological invariance of the Witten index, J. Funct. Anal. 79, 91-102 (1988).

[19] F. GesZTesy AND M. Zinchenko, Symmetrized perturbation determinants and applications to boundary data maps and Krein-type resolvent formulas, Proc. London Math. Soc. (3) 104, 577-612 (2012).

[20] I. Gohberg And M. G. Krein, Introduction to the Theory of Linear Nonselfadjoint Operators, Translations of Mathematical Monographs, Vol. 18, Amer. Math. Soc., Providence, RI, 1969.

[21] T. KATO, Perturbation Theory for Linear Operators, corr. printing of the 2nd ed., Springer, Berlin, 1980.

[22] A. Pushnitski, The spectral flow, the Fredholm index, and the spectral shift function, in Spectral Theory of Differential Operators: M. Sh. Birman 80th Anniversary Collection, T. Suslina and D. Yafaev (eds.), AMS Translations, Ser. 2, Advances in the Mathematical Sciences, Vol. 225, Amer. Math. Soc., Providence, RI, 2008, pp. 141-155.

[23] M. Reed And B. Simon, Methods of Modern Mathematical Physics. I: Functional Analysis, revised and enlarged edition, Academic Press, New York, 1980.

[24] J. Robbin And D. Salamon, The spectral flow and the Maslov index, Bull. London Math. Soc. 27, 1-33 (1995).

[25] B. Simon, Trace Ideals and Their Applications, 2nd ed., Mathematical Surveys and Monographs, Vol. 120, Amer. Math. Soc., Providence, RI, 2005.

[26] J. Weidmann, Linear Operators in Hilbert Spaces, Graduate Texts in Mathematics, Vol. 68, Springer, New York, 1980.

[27] K. P. Wojciechows KI, Spectral shift function and eta function. Elementary Example, unpublished manuscript, March, 2007, kindly communicated to us by M. Lesch. 
[28] D. R. Yafaev, Mathematical Scattering Theory. General Theory, Amer. Math. Soc., Providence, RI, 1992. 